medRxiv preprint doi: https://doi.org/10.1101/2020.09.23.20195719; this version posted September 24, 2020. The copyright holder for this preprint (which was not certified by peer review) is the author/funder, who has granted medRxiv a license to display the preprint in perpetuity.

It is made available under a CC-BY-NC-ND 4.0 International license .

\title{
Feasibility of generalised DKI approach for brain glioma grading
}

E.L. Pogosbekian ${ }^{1,2}$, I.N. Pronin ${ }^{1}$, N.E. Zakharova ${ }^{1}$, A.I. Batalov ${ }^{1}$, A.M. Turkin ${ }^{1}$, T.A. Konakova ${ }^{1}$, I.I. Maximov $3,4, \dagger$

${ }^{1}$ Neuroimaging Department, N.N. Burdenko National Medical Research Centre of Neurosurgery, Moscow, Russian Federation

2 General and Clinical Neurophysiology Lab, Institute of Higher Nervous Activity and Neurophysiology of RAS, Moscow, Russian Federation

${ }^{3}$ Department of Psychology, University of Oslo, and Norwegian Centre for Mental Disorders Research (NORMENT), Department of Mental Health and Addiction, Oslo University Hospital, Oslo, Norway

${ }^{4}$ Department of Health and Functioning, Western Norway University of Applied Sciences, Bergen, Norway

Number of Figures: 6

Number of Tables: 3

Number of words:

$\dagger$ corresponding authors:

I.I. Maximov: ivan.maximov@psykologi.uio.no

Department of Psychology, University of Oslo, Oslo, Norway 
medRxiv preprint doi: https://doi.org/10.1101/2020.09.23.20195719; this version posted September 24, 2020. The copyright holder for this

preprint (which was not certified by peer review) is the author/funder, who has granted medRxiv a license to display the preprint in perpetuity.

It is made available under a CC-BY-NC-ND 4.0 International license .

\begin{abstract}
Purpose: An accurate differentiation of brain glioma grade constitutes an important clinical issue. Powerful non-invasive approach based on diffusion MRI has already demonstrated its feasibility in glioma grade stratification. However, the conventional diffusion tensor (DTI) and kurtosis imaging (DKI) demonstrated moderate sensitivity and performance in glioma grading. In the present work, we apply generalised DKI (gDKI) approach in order to assess its diagnostic accuracy and potential application in glioma grading.

Methods: Diffusion scalar metrics were obtained from 50 patients with different glioma grades confirmed by histological tests following biopsy or surgery. All patients were divided into two groups with low- and high-grade gliomas as II grade versus III and IV grades, respectively. For a comparison, trained radiologists segmented the brain tissue into three regions with solid tumour, oedema, and normal appearing white matter. For each region we estimated the conventional and gDKI metrics including DTI maps.
\end{abstract}

Results: We found high correlations between DKI and gDKI metrics in high-grade glioma. Further, gDKI metrics enabled introduction of a complementary measure for glioma differentiation based on correlations between the conventional and generalised approaches. Both conventional and generalised DKI metrics showed quantitative maps of tumour heterogeneity and oedema behaviour. gDKI approach demonstrated largely similar sensitivity and specificity in low-high glioma differentiation as in the case of conventional DKI method.

Conclusion: The generalised diffusion kurtosis imaging enables differentiation of low and high grade gliomas at the same level as the conventional DKI. Additionally, gDKI exhibited higher tissue contrast between tumour and healthy tissue and, thus, may contribute as a complementary source of information on tumour heterogeneity.

Keywords: glioblastoma multiform; glioma discrimination; diffusion kurtosis imaging

\title{
Declaration
}

Funding: This work was partially supported by Research Council of Norway (249795).

Conflict of interests: Authors declare that they have no conflict of interests.

Ethical approval: All procedures performed in study involving human participants were in accordance with the ethical standards of the institutional and/or national research committee and with the 1964 Helsinki declaration and its later amendments or comparable ethical standards.

Informed consent: Informed consent was obtained from all individual participants included in the study. 
medRxiv preprint doi: https://doi.org/10.1101/2020.09.23.20195719; this version posted September 24, 2020. The copyright holder for this

preprint (which was not certified by peer review) is the author/funder, who has granted medRxiv a license to display the preprint in perpetuity.

It is made available under a CC-BY-NC-ND 4.0 International license .

\section{Introduction}

Primary brain glioma is a highly widespread type of intra-axial brain tumours seen approximately in one fourth diagnosed tumour cases [1]. Classification of the brain tumours is performed in accordance with grades introduced by the World Health Organisation (WHO) [2] which combine histopathological and molecular features into integrated tumour characterisation. As a result, glioma grades are ordered from I up to IV grade related to tumour's aggressiveness and malignancy. Both the tumour's diagnosis and its grade can be reliably confirmed by a histopathologic analysis of the tumour's tissue obtained from an invasive procedure such as biopsy or surgery. However, non-invasive assessments of the tumour's malignancy are necessary for clinical treatment, surgery planning and survival rate estimations. In particular, this applies to low-grade tumour cases where such assessments contribute to treatment efficacy and, as a result, improve quality of life for patients. Magnetic resonance imaging (MRI) offers a wide spectrum of tissue visualisation approaches for clinical purposes allowing one to detect and localise brain's abnormalities with a certain degree of specificity. Nevertheless, conventional MRI techniques such as structural $\mathrm{T}_{1} / \mathrm{T}_{2}$-weighted imaging with or without contrast agents or magnetic resonance spectroscopy demonstrate limited sensitivity and specificity for brain glioma differentiation [3], [4].

Diffusion weighted imaging (DWI) has exhibited its superiority as a non-invasive brain imaging technique and has been applied in a plethora of clinical settings [5], [6], [7]. Diffusion tensor imaging (DTI) [8], the most often used DWI approach, has also been utilised for tumour assessment by many research groups [9], [10], [11]. In turn, diffusion kurtosis imaging (DKI) [12] is a powerful extension of the conventional DTI technique enabling estimation of the degree of non-Gaussian diffusion in the brain tissue by estimating higher order cumulant of the diffusion signal expansion [13]. The deviation of water molecule diffusion from free diffusion behaviour or Gaussian distribution is caused by complex organisation of the brain tissue, where cellular and neurite barriers alter the probability distribution of water diffusion. This phenomenon is particularly important for the brain tumour detection and assessment due to increased tissue complexity resulting from the high cancer cell proliferation rate, increased tumour tissue vascularisation, presence of oedema as well as necrosis. DKI is extensively used in brain imaging [14], [15], [16], in particular, for tumour differentiation [17], [18], [19], [20], [21]. Despite the fact that DKI demonstrated quite promising results in the case of glioma grading, the kurtosis scalar metrics such as mean kurtosis, still lack the accurate and reliable glioma 
grading in the case of both low- (i.e. glioma-I and II) and high- (glioma-III and IV) grade gliomas [18], [22], [23].

In the present study, we adapted the generalised kurtosis approach (GK) [24] for glioma differentiation in order to evaluate as well as to add a complementary information and, thus, increase the accuracy of glioma grading. An advantage of the third order term of cumulant expansion of the diffusion signal has been demonstrated for differentiation of low- and high-grade gliomas together with an application of the conventional kurtosis (CK) approach. In order to emphasise the advantage of the DKI imaging for glioma grading, we concurrently applied both DKI and DTI metrics derived from the $\mathrm{CK}$ and GK approaches. Hence, the purpose of the present study was to investigate the value of the generalised DKI metrics for the evaluation of glioma grading.

\section{Materials and Methods}

\section{Patients}

50 adult patients (mean age: $44 \pm 13$ years, minimal age: 24 years, maximal age: 70 years; male/female ratio: 30/20) initially diagnosed with glioma were recruited at our Institution and examined with MRI prior to biopsy, surgery, radiation or chemotherapy treatments. The diagnosis of glioma and WHO grade [25] were confirmed by histological and immunohistochemical examinations for each subject following MRI examination.

The study included 19 patients with glioma grade II, 8 patients with glioma grade III, and 23 patients with glioma grade IV. The group of patients with glioma-II consisted of 6 subjects with oligoastrocytoma, 1 subject with oligodendroglioma, 1 subject with hemistocytic astrocytoma, 11 subjects with diffuse astrocytoma. The group of patients with glioma-III consisted of 7 subjects with anaplastic astrocytoma, 1 subject with anaplastic oligoastrocytoma. The group of patients with gliomaIV consisted of the 22 subjects with glioblastoma multiform and 1 subject with gliosarkoma.

\section{Imaging}

All patients underwent MRI examination with a 3T GE scanner (SignaHDxt) using a 8-channel phased-array head coil. The imaging protocol included precontrast $\mathrm{T}_{2}$-weighted FLAIR and diffusionweighted sequences, followed by postcontrast $\mathrm{T}_{1}$-weighted imaging with a gadolinium based contrast agent. The parameters specific to anatomical imaging sequences were the following: $\mathrm{T}_{1}$-weighted: FOV $=240 \mathrm{~mm}^{2}, \mathrm{TE} / \mathrm{TR}=3500 / 8800 \mathrm{~ms}$, resolution $1 \mathrm{~mm}^{3} ; \mathrm{T}_{2}$-weighted FLAIR: FOV = $240 \mathrm{~mm}^{2}$, TE/TR $=120 / 9500 \mathrm{~ms}$, resolution $1 \times 1 \times 5 \mathrm{~mm}^{3}$; diffusion-weighted imaging: $\mathrm{FOV}=240 \mathrm{~mm}^{2}, \mathrm{TE} / \mathrm{TR}=$ 
$102.8 / 10000 \mathrm{~ms}$, resolution $3 \mathrm{~mm}^{3}, b$-values $=0,1000$, and $2500 \mathrm{~s} / \mathrm{mm}^{2}$ with 60 encoding diffusion directions, directions were non-coplanar uniformly distributed over the unit sphere. The diffusion imaging was obtained using a single-shot echo echo planar imaging sequence.

\section{Image Analysis}

Prior to estimation of scalar diffusion metrics, the raw diffusion data were corrected for image distortions in accordance with optimised pipeline [26], i.e. for noise [27] and Gibbs-ringing distortions [28], eddy-current distortions including bulk head motion artefacts were corrected using eddy utility from FSL [29], [30], [31]. Conventional DKI metrics such as mean, axial, radial kurtosis, and kurtosis anisotropy (MK, AK, RK and KA, respectively) were estimated using an iterative weighted least squares approach [32] and supplied by the complementary DTI measures known as fraction anisotropy (FA), mean diffusivity (MD), axial diffusivity (AD), and radial diffusivity (RD). The conventional DKI metrics were obtained from the second order cumulant expansion in the following form [12]:

$$
\ln [S(b)]=\ln \left[S_{0}\right]-b D+b^{2} D^{2} \frac{K}{6}=F_{1}
$$

where $b$ is the diffusion weighting, $D$ is the diffusion coefficient, $S_{0}$ is the signal without the diffusion weightings, and $K$ is the diffusion kurtosis. The righthand expression is defined as $F_{1}$. The generalised DKI parameters were estimated using in-house MATLAB scripts (The MathWorks, Natick, MA USA) including the third order terms [24]:

$$
\ln [S(b, a)]=F_{1}+(a-1) \sqcap D^{3} b^{3} \frac{K^{2}}{54},
$$

where $\alpha$ is the adjusting variable. In the case of $\alpha=1$, the last equation leads to the conventional DKI expression $F_{1}$. In the present work, we used an optimised $\alpha$ value at 2/7 [24]. Subsequently, the estimated diffusion scalar maps for both cases of conventional and generalised kurtosis were aligned and interpolated to $\mathrm{T}_{1}$-weighted anatomical image for each patient using the cubic spline interpolation algorithm and affine transformation. Two independent radiologists manually delineated three regions of interest for each subject: tumour, oedema and normal appearing white matter (NAWM) using 3 MRI contrasts: $\mathrm{T}_{1}$-weighted, $\mathrm{T}_{2}$-weighted, and MD map estimated from CK approach. Examples of images for low- and high-grade gliomas with the 3 aforementioned regions of interest are presented in Figures 1 and 2 , respectively.

\section{Statistical analysis}

Statistical analyses were performed using $R$ Project (http://r-project.org) and in-house MATLAB scripts. Some voxels among the patient maps contained of corrupted diffusion metrics derived from the 
DTI and DKI tensors. In order to prevent any statistical bias in analysis, we excluded such voxels from consideration using inequalities: $\mathrm{MK}<0$ and $\mathrm{MK}>3$, for both $\mathrm{CK}$ and GK approaches. Mean values and standard deviations of all diffusion metrics were calculated from the tumour, oedema and NAWM regions. These values were used for the low- and high-grade glioma differentiation and assessed with a Mann-Whitney-Wilcox U-test with statistical significance reported at the 0.05 level. Effect sizes were calculated with Cohen's $d$ in order to estimate the magnitude of the differences between the conditions/groups. Correlations between the conventional and generalised DKI metrics have been estimated using a linear regression model: $y=k_{0}+k_{1} \bullet x$, and package stat from $R$ Project. Receiver operation characteristic (ROC) analysis was performed using package $p R O C$ in order to compute the area under curve (AUC) for estimation of the diffusion model performance for tumour differentiation. The squared Pearson correlation coefficients were estimated using the package stat from $R$ Project.

\section{Results}

Figure 1 and 2 are visual examples of manual masks of three regions, i.e. tumour, oedema, and NAWM together with examples of MD and MK maps for the low- and high-grade glioma patients, estimated by the CK and GK approaches. All maps are presented in $\mathrm{T}_{1}$-weighted space with a spatial interpolation of diffusion maps. Briefly, we can see that the visual quality of MD maps between CK and GK approaches is very similar. In turn, as already was shown in [24], the tissue contrast for DKI maps, e.g. MK, is higher for GK approach. Squared outlier voxels both in Figs. 1 and 2 are the result of fitting problems due to noisy diffusion signal. These voxels have been excluded from the analysis.

Figures 3 and 4 present the correlations of diffusion metrics obtained by CK and GK signal expansions. Figure 3 shows DTI metrics only, i.e. FA, MD, AD, and RD with corresponding R-squared values which in the case of linear correlations coincide with the squared Pearson correlation coefficients (see Table 1). In Fig. 4 we present DKI metrics only, i.e. KA, MK, AK, and RK with corresponding R-squared values (see Tab. 1). The scatter plots were estimated voxelwisely for the tumour masks only from low- and high-grade gliomas, respectively. In short, linear correlations between CK and GK estimations are higher for the high-grade glioma in contrast to the low grade glioma. This effect is present for all DTI metrics. In the case of DKI metrics, it applies only for KA and AK metrics. Interestingly, MK and RK metrics have two linearly dependent subgroups of voxels (see, for example, Fig. 4: LGG), which merge as glioma malignancy increases (see, Fig. 4: HGG). 
In Figure 5 we present boxplots of the mask-averaged diffusion metrics estimated from CK and GK approaches. The averages were performed for each ROI such as tumour, oedema, and NAWM for each patient. The boxplots are structured as ROI-wise and glioma grades pairs. For each pair of lowand high-grade gliomas, we performed the Mann-Whitney-Wilcox U-test in order to check for significant $(\mathrm{p}<0.05)$ differences between glioma grades in all regions including tumour, oedema and NAWM. There were no significant differences between low- and high-grade glioma diffusion metrics for oedema, and there was difference between GK metrics of RD for NAWM. However, for tumour regions, both $\mathrm{CK}$ and GK metrics except for KA and FA, we revealed a significant difference for lowand high-grade gliomas (marked by asterisk in Fig. 5). In the case of FA metrics, GK approach did not reveal significant differences between glioma grades. The effect sizes for the diffusion metric differences detected by both CK and GK approaches are summarised in Table 2. For DTI metrics, the effect size is large for both kurtosis approaches, while for DKI metrics the differentiation possesses very large effect size except for GK-derived RK metric. We also performed a pair comparison between $\mathrm{CK}$ and GK metrics marked by the cyan background colour for significant differences $(\mathrm{p}<0.05)$ between the CK-GK metrics. Interestingly, for all DKI metrics and ROIs, there were significant difference between CK and GK metrics. However, for DTI metrics, the significant difference was detected only for NAWM regions for FA, MD, and RD metrics.

It is of vital importance to perform a comparison between $\mathrm{CK}$ and $\mathrm{GK}$ approaches in terms of their sensitivity and specificity. These results are presented in Figure 6. Both approaches demonstrated high rate of sensitivity and specificity for low and high glioma discrimination with negligible difference between CK and GK approaches. The estimated sensitivity, specificity, cutoff and area under curves (AUC) are summarised in Table 3.

\section{Discussion}

In the present study we estimated two diffusion imaging approaches based on the conventional and generalised kurtosis expansions. The scalar metrics based on GK approach demonstrated higher linear correlations with CK derived metrics for high-grade glioma and comparable sensitivity and specificity in glioma differentiation between low- and high-grade gliomas. In turn, the scalar diffusion metrics based on GK approach exhibited brighter tumour and peritumoural oedema contrasts compared to healthy tissue allowing clinicians to better visualise tumour spreading in a healthy brain tissue environment. 
The tumour grade differentiation constitutes a challenge for many imaging techniques such as MRI, CT, or PET. This is due to the need of a quantitative parametrisation enabling both the assessment of damage of healthy brain tissue by aggressive growth of tumour and the degree of the malignancy of this process. This is particularly complicated due to multifarous forms of tumour manifestation, including cell swelling, microvasculature proliferation, tumour heterogeneity, presence of necrosis. Thus, the diffusion MR imaging grants two levels of tumour assessment, that is easy-to-interpret scalar maps and sensitivity of these maps to the micrometer scale of tissue changes. Therefore, diffusion kurtosis imaging offers an excellent tool for probing brain tumour, enabling estimation of the qualitative and quantitative differences between different glioma grades and their dynamics.

The difference between the conventional and generalised kurtosis estimations is defined by a fraction multiplyer $\alpha$ and a quadratic kurtosis term in Eq. (2). It is interesting that dispersion in the distribution between DTI metrics (see Fig. 3) is quite low and does not depend on glioma grade. However, in the case of kurtosis scalar maps (see Fig. 4), in particular for MK and RK, there is a stronger value spreading in low glioma metric correlations. Such kind of distribution behaviour can indicate two different types of tissue organisation present at early stages of the glioma tumour: resting healthy brain tissue and highly heterogeneous tumour. As glioma malignancy increases, the heterogeneous tumour tissue becomes dominant due to aggressive growth which leads to higher correlations between CK and GK metrics, i.e. quadratic kurtosis term might drive this effect. We see that GK-derived MK and RK metrics are more sensitive to the tumour heterogeneity in low-grade glioma. Therefore, a variation in data dispersion between CK and GK metrics, in particular, in kurtosis metrics, might provide us with an additional information about glioma grades. Notably, this information is accessible only for voxelwise assessment, where typical glioma grade differentiation is performed for region-averaged diffusion metrics.

This hypothesis is supported by significant differences between CK and GK metrics for kurtosis metrics in contrast to the DTI ones (see Fig. 5). For kurtosis metrics, the significant differences between $\mathrm{CK}$ and GK derived values were found in all regions: tumour, oedema, and NAWM. On the other hand, DTI metrics demonstrated significant differences only for normally appearing brain tissue, likely due to the different regions of the brain chosen as NAWM and strong age dependence of the patients. Thus, the quadratic kurtosis term strongly influences the kurtosis scalar metrics while it does not affect the DTI ones. 
It is quite important to estimate general brain changes associated with tumour grades in accordance with healthy tissue. All DTI metrics (FA, MD, AD, and RD) demonstrated their potential as a glioma grade biomarker, except for FA maps derived from GK (see Tabs. 2 and 3). The values of MD, AD, and RD show lower diffusivities in high grade glioma for both CK and GK approaches. In turn, diffusion metrics for both low- and high-grade gliomas demonstrated higher diffusivities in contrast to the NAWM values. This effect might be related to damage of healthy tissue as extra-cellular space increases accompanied by simultaneous microvascularisation process in the tumour tissue. Interestingly, low-grade glioma tissue retains higher diffusivities in contrast to high grade one, likely due to an increased cell proliferation rate in high-grade glioma, i.e. emergence of more cellular barriers limiting the water diffusion.

Kurtosis scalar maps exhibited the same trends as DTI metrics for low and high glioma differentiation and the contrast between the tumour and healthy tissue regions. Notably, KA did not reveal significant differences between low- and high-grade gliomas. Nonetheless, MK, AK, and RK metrics demonstrated lower kurtosis values in low-grade glioma in contrast to both high-grade glioma and healthy tissue (see Fig. 5).

We did not find significant differences for oedema regions for neither low nor high grade gliomas. At the same time, oedema metrics exhibited close range of kurtosis and DTI values with high grade glioma, suggesting that the peritumoural tissue organisation is more complex than free water diffusion. This effect may be caused by the compression of the surrounding cells and resting microstructure of healthy tissue. However, this hypothesis should be verified and examined with different types of glioma spreading processes such as the level of cell infiltration that demands high resolution diffusion measurements.

As a limitation of the study we should note not an optimal choice of adjusting variable $\alpha$. A more accurate model fitted to the tumour microstructure [33] might increase feasibility of GK approach and its performance in the case of glioma grading. Addtionally, advanced diffusion techniques including fast kurtosis [34], isotropic diffusion weighting [35], [36], [37] and multidimensional diffusion imaging [38], [39] might help clinicians to perform a robust, fast and non-invasive glioma differentiation. We plan to implement and apply these approaches for research and patiant treatment in the future.

In conclusion, the generalised diffusion kurtosis imaging presents an additional source of information enabling differentiation of low- and high-grade gliomas at the same level as the 
medRxiv preprint doi: https://doi.org/10.1101/2020.09.23.20195719; this version posted September 24, 2020. The copyright holder for this preprint (which was not certified by peer review) is the author/funder, who has granted medRxiv a license to display the preprint in perpetuity.

It is made available under a CC-BY-NC-ND 4.0 International license .

conventional DKI. The GK approach exhibited higher tissue contrast and, thus, offers more sensitive scalar maps to glioma tissue heterogeneity. 


\section{References}

[1] Q. T. Ostrom, H. Gittleman, G. Truitt, A. Boscia, C. Kruchko, and J. S. Barnholtz-Sloan, “CBTRUS Statistical Report: Primary Brain and Other Central Nervous System Tumors Diagnosed in the United States in 2011-2015," Neuro-Oncology, vol. 20, no. suppl_4, pp. iv1-iv86, Oct. 2018, doi: 10.1093/neuonc/noy131.

[2] P. Wesseling and D. Capper, "WHO 2016 Classification of gliomas," Neuropathology and Applied Neurobiology, vol. 44, no. 2, pp. 139-150, Feb. 2018, doi: 10.1111/nan.12432.

[3] N. Upadhyay and A. D. Waldman, "Conventional MRI evaluation of gliomas," The British Journal of Radiology, vol. 84, no. special_issue_2, pp. S107-S111, Dec. 2011, doi: 10.1259/bjr/65711810.

[4] Q. Wang et al., "The diagnostic performance of magnetic resonance spectroscopy in differentiating high-from low-grade gliomas: A systematic review and meta-analysis," European Radiology, vol. 26, no. 8, pp. 2670-2684, Aug. 2016, doi: 10.1007/s00330-015-4046-z.

[5] J. Puig et al., "Diffusion tensor imaging as a prognostic biomarker for motor recovery and rehabilitation after stroke," Neuroradiology, vol. 59, no. 4, pp. 343-351, Apr. 2017, doi: 10.1007/s00234-017-1816-0.

[6] C. Andica et al., "MR biomarkers of degenerative brain disorders derived from diffusion imaging," Journal of Magnetic Resonance Imaging, Dec. 2019, doi: 10.1002/jmri.27019.

[7] E. I. Kremneva et al., "Feasibility of Non-Gaussian Diffusion Metrics in Chronic Disorders of Consciousness," Brain Sciences, vol. 9, no. 5, p. 123, May 2019, doi: 10.3390/brainsci9050123.

[8] P. J. Basser, J. Mattiello, and D. LeBihan, "MR diffusion tensor spectroscopy and imaging," Biophysical Journal, vol. 66, no. 1, pp. 259-267, Jan. 1994, doi: 10.1016/S0006-3495(94)80775-1.

[9] R. Jiang, F.-Z. Du, C. He, M. Gu, Z.-W. Ke, and J.-H. Li, “The Value of Diffusion Tensor Imaging in Differentiating High-Grade Gliomas from Brain Metastases: A Systematic Review and MetaAnalysis," PLoS ONE, vol. 9, no. 11, p. e112550, Nov. 2014, doi: 10.1371/journal.pone.0112550.

[10]P. Svolos et al., "The role of diffusion and perfusion weighted imaging in the differential diagnosis of cerebral tumors: a review and future perspectives," Cancer Imaging, vol. 14, no. 1, Dec. 2014, doi: 10.1186/1470-7330-14-20.

[11]C. H. Suh, H. S. Kim, S. C. Jung, and S. J. Kim, "Diffusion-Weighted Imaging and Diffusion Tensor Imaging for Differentiating High-Grade Glioma from Solitary Brain Metastasis: A Systematic Review and Meta-Analysis," American Journal of Neuroradiology, vol. 39, no. 7, pp. 1208-1214, Jul. 2018, doi: 10.3174/ajnr.A5650.

[12]J. H. Jensen, J. A. Helpern, A. Ramani, H. Lu, and K. Kaczynski, "Diffusional kurtosis imaging: The quantification of non-gaussian water diffusion by means of magnetic resonance imaging," Magnetic Resonance in Medicine, vol. 53, no. 6, pp. 1432-1440, Jun. 2005, doi: 10.1002/mrm.20508.

[13]V. G. Kiselev, "Fundamentals of diffusion MRI physics: Fundamentals of Diffusion MRI Physics," NMR in Biomedicine, vol. 30, no. 3, p. e3602, Mar. 2017, doi: 10.1002/nbm.3602.

[14]J. H. Jensen and J. A. Helpern, "MRI quantification of non-Gaussian water diffusion by kurtosis analysis," NMR in Biomedicine, vol. 23, no. 7, pp. 698-710, May 2010, doi: 10.1002/nbm.1518.

[15]A. B. Rosenkrantz et al., "Body diffusion kurtosis imaging: Basic principles, applications, and considerations for clinical practice: Body Diffusion Kurtosis Imaging," Journal of Magnetic Resonance Imaging, vol. 42, no. 5, pp. 1190-1202, Nov. 2015, doi: 10.1002/jmri.24985.

[16]F. Grinberg et al., "Diffusion kurtosis metrics as biomarkers of microstructural development: A comparative study of a group of children and a group of adults," NeuroImage, vol. 144, pp. 12-22, Jan. 2017, doi: 10.1016/j.neuroimage.2016.08.033. 
[17]J.-M. Hempel et al., "Histogram analysis of diffusion kurtosis imaging estimates for in vivo assessment of 2016 WHO glioma grades: A cross-sectional observational study," European Journal of Radiology, vol. 95, pp. 202-211, Oct. 2017, doi: 10.1016/j.ejrad.2017.08.008.

[18]A. Tietze et al., "Mean Diffusional Kurtosis in Patients with Glioma: Initial Results with a Fast Imaging Method in a Clinical Setting," American Journal of Neuroradiology, vol. 36, no. 8, pp. 1472-1478, Aug. 2015, doi: 10.3174/ajnr.A4311.

[19]P. Raab, E. Hattingen, K. Franz, F. E. Zanella, and H. Lanfermann, "Cerebral Gliomas: Diffusional Kurtosis Imaging Analysis of Microstructural Differences," Radiology, vol. 254, no. 3, pp. 876881, Mar. 2010, doi: 10.1148/radiol.09090819.

[20]A. Falk Delgado, M. Nilsson, D. van Westen, and A. Falk Delgado, "Glioma Grade Discrimination with MR Diffusion Kurtosis Imaging: A Meta-Analysis of Diagnostic Accuracy," Radiology, vol. 287, no. 1, pp. 119-127, Apr. 2018, doi: 10.1148/radiol.2017171315.

[21]I. I. Maximov, A. S. Tonoyan, and I. N. Pronin, "Differentiation of glioma malignancy grade using diffusion MRI,” Physica Medica, vol. 40, pp. 24-32, Aug. 2017, doi: 10.1016/j.ejmp.2017.07.002.

[22]X.-X. Qi et al., "Histogram analysis of diffusion kurtosis imaging derived maps may distinguish between low and high grade gliomas before surgery," European Radiology, vol. 28, no. 4, pp. 1748-1755, Apr. 2018, doi: 10.1007/s00330-017-5108-1.

[23]S. Vellmer, A. S. Tonoyan, D. Suter, I. N. Pronin, and I. I. Maximov, "Validation of DWI preprocessing procedures for reliable differentiation between human brain gliomas," Zeitschrift für Medizinische Physik, vol. 28, no. 1, pp. 14-24, Feb. 2018, doi: 10.1016/j.zemedi.2017.04.005.

[24]J. H. Jensen, V. Mohanty, E. T. McKinnon, and J. A. Helpern, "Optimizing the Signal Model for Diffusional Kurtosis Imaging," in Proceedings of International Society of Magnetic Resonance in Medicine, 2017, p. 1731, [Online]. Available: http://indexsmart.mirasmart.com/ISMRM2017/PDFfiles/1731.html.

[25]S. Feiden and W. Feiden, "WHO-Klassifikation der ZNS-Tumoren: Revidierte Fassung von 2007 mit kritischen Anmerkungen zum „Typing“ und „Grading“ diffuser Gliome,” Der Pathologe, vol. 29, no. 6, pp. 411-421, Nov. 2008, doi: 10.1007/s00292-008-1064-5.

[26]I. I. Maximov, D. Alnæs, and L. T. Westlye, “Towards an optimised processing pipeline for diffusion magnetic resonance imaging data: Effects of artefact corrections on diffusion metrics and their age associations in UK Biobank," Human Brain Mapping, vol. 40, no. 14, pp. 4146-4162, Oct. 2019, doi: 10.1002/hbm.24691.

[27]J. Veraart, D. S. Novikov, D. Christiaens, B. Ades-aron, J. Sijbers, and E. Fieremans, "Denoising of diffusion MRI using random matrix theory," NeuroImage, vol. 142, pp. 394-406, Nov. 2016, doi: 10.1016/j.neuroimage.2016.08.016.

[28]E. Kellner, B. Dhital, V. G. Kiselev, and M. Reisert, "Gibbs-ringing artifact removal based on local subvoxel-shifts: Gibbs-Ringing Artifact Removal," Magnetic Resonance in Medicine, vol. 76, no. 5, pp. 1574-1581, Nov. 2016, doi: 10.1002/mrm.26054.

[29]M. Jenkinson, C. F. Beckmann, T. E. J. Behrens, M. W. Woolrich, and S. M. Smith, "FSL," NeuroImage, vol. 62, no. 2, pp. 782-790, Aug. 2012, doi: 10.1016/j.neuroimage.2011.09.015.

[30]J. L. R. Andersson, S. Skare, and J. Ashburner, "How to correct susceptibility distortions in spinecho echo-planar images: application to diffusion tensor imaging," NeuroImage, vol. 20, no. 2, pp. 870-888, Oct. 2003, doi: 10.1016/S1053-8119(03)00336-7.

[31]J. L. R. Andersson and S. N. Sotiropoulos, "An integrated approach to correction for off-resonance effects and subject movement in diffusion MR imaging," NeuroImage, vol. 125, pp. 1063-1078, Jan. 2016, doi: 10.1016/j.neuroimage.2015.10.019. 
medRxiv preprint doi: https://doi.org/10.1101/2020.09.23.20195719; this version posted September 24, 2020. The copyright holder for this

preprint (which was not certified by peer review) is the author/funder, who has granted medRxiv a license to display the preprint in perpetuity.

It is made available under a CC-BY-NC-ND 4.0 International license .

[32]J. Veraart, J. Sijbers, S. Sunaert, A. Leemans, and B. Jeurissen, "Weighted linear least squares estimation of diffusion MRI parameters: Strengths, limitations, and pitfalls," NeuroImage, vol. 81, pp. 335-346, Nov. 2013, doi: 10.1016/j.neuroimage.2013.05.028.

[33]F. Zaccagna et al., "Non-invasive assessment of glioma microstructure using VERDICT MRI: correlation with histology," European Radiology, vol. 29, no. 10, pp. 5559-5566, Oct. 2019, doi: 10.1007/s00330-019-6011-8.

[34]B. Hansen, T. E. Lund, R. Sangill, E. Stubbe, J. Finsterbusch, and S. N. Jespersen, "Experimental considerations for fast kurtosis imaging: Experimental Considerations for Fast Kurtosis Imaging," Magnetic Resonance in Medicine, vol. 76, no. 5, pp. 1455-1468, Nov. 2016, doi: 10.1002/mrm.26055.

[35]S. Eriksson, S. Lasic, and D. Topgaard, "Isotropic diffusion weighting in PGSE NMR by magicangle spinning of the q-vector," Journal of Magnetic Resonance, vol. 226, pp. 13-18, Jan. 2013, doi: 10.1016/j.jmr.2012.10.015.

[36]S. Vellmer, R. Stirnberg, D. Edelhoff, D. Suter, T. Stöcker, and I. I. Maximov, "Comparative analysis of isotropic diffusion weighted imaging sequences," Journal of Magnetic Resonance, vol. 275, pp. 137-147, Feb. 2017, doi: 10.1016/j.jmr.2016.12.011.

[37]I. I. Maximov and S. Vellmer, "Isotropically weighted intravoxel incoherent motion brain imaging at 7T," Magnetic Resonance Imaging, vol. 57, pp. 124-132, Apr. 2019, doi: 10.1016/j.mri.2018.11.007.

[38]D. Topgaard, "Multidimensional diffusion MRI," Journal of Magnetic Resonance, vol. 275, pp. 98-113, Feb. 2017, doi: 10.1016/j.jmr.2016.12.007.

[39]C.-F. Westin et al., "Q-space trajectory imaging for multidimensional diffusion MRI of the human brain," NeuroImage, vol. 135, pp. 345-362, Jul. 2016, doi: 10.1016/j.neuroimage.2016.02.039. 
medRxiv preprint doi: https://doi.org/10.1101/2020.09.23.20195719; this version posted September 24, 2020. The copyright holder for this preprint (which was not certified by peer review) is the author/funder, who has granted medRxiv a license to display the preprint in perpetuity.

It is made available under a CC-BY-NC-ND 4.0 International license .

Table 1 The results for linear correlations between diffusion metrics obtained from CK and GK approaches (see Figs. 3 and 4). The linear model is $y=b_{0}+b_{1} \cdot x$, R-square is the coefficient of determination also coinciding with the squared Pearson correlation coefficient. LGG - low grade glioma, HGG - high grade glioma.

\begin{tabular}{|c|c|c|c|c|c|c|c|c|}
\hline LGG & FA & MD & AD & RD & KA & MK & AK & RK \\
\hline $\begin{array}{c}\text { intercept } \\
b_{0}\end{array}$ & 0.053 & -0.017 & 0.091 & 0.025 & 0.19 & -0.12 & 0.0088 & -0.15 \\
\hline slope $b_{1}$ & 0.73 & 1.1 & 1.0 & 1.0 & 0.88 & 1.7 & 1.7 & 1.6 \\
\hline R-square & 0.693 & 0.981 & 0.906 & 0.97 & 0.463 & 0.944 & 0.777 & 0.942 \\
\hline HGG & 0.028 & 0.022 & 0.054 & 0.028 & 0.14 & -0.1 & -0.018 & -0.13 \\
\hline $\begin{array}{c}\text { intercept } \\
b_{0}\end{array}$ & 0.85 & 1.0 & 1.0 & 1.0 & 0.93 & 1.6 & 1.7 & 1.6 \\
\hline slope $b_{1}$ & 0.814 & 0.977 & 0.953 & 0.97 & 0.756 & 0.889 & 0.804 & 0.855 \\
\hline R-square & 0.87 & & & & & & \\
\hline
\end{tabular}

Table 2 The effect size of significant differences between low and high grade gliomas detected by CK and GK derived diffusion metrics (see Fig. 5). The effect size is estimated using the Cohen's $d$.

\begin{tabular}{|c|c|c|c|c|c|c|c|c|}
\hline $\begin{array}{c}\text { Effect } \\
\text { size }\end{array}$ & FA & MD & AD & RD & KA & MK & AK & RK \\
\hline CK & 0.99 & 1.21 & 1.11 & 1.23 & 0.11 & 1.56 & 1.47 & 1.52 \\
\hline GK & 0.65 & 1.15 & 1.13 & 1.15 & 0.30 & 1.50 & 1.35 & 0.24 \\
\hline
\end{tabular}


medRxiv preprint doi: https://doi.org/10.1101/2020.09.23.20195719; this version posted September 24, 2020. The copyright holder for this preprint (which was not certified by peer review) is the author/funder, who has granted medRxiv a license to display the preprint in perpetuity.

It is made available under a CC-BY-NC-ND 4.0 International license .

Table 3 Differentiation performance for diffusion metrics estimated by CK and GK approaches. AUC of ROC (see Fig. 6), cutoff, senstivity and specificity values are presented.

\begin{tabular}{|c|c|c|c|c|c|c|c|c|}
\hline CK & FA & MD & AD & RD & $\mathbf{K A}$ & MK & $\mathbf{A K}$ & RK \\
\hline AUC & 0.77 & 0.79 & 0.78 & 0.80 & 0.53 & 0.87 & 0.86 & 0.86 \\
\hline cutoff & 0.15 & 1.50 & 1.72 & 1.39 & 0.23 & 0.53 & 0.41 & 0.54 \\
\hline sensitivity & 0.74 & 0.71 & 0.71 & 0.71 & 0.48 & 0.74 & 0.93 & 0.74 \\
\hline specificity & 0.74 & 0.84 & 0.84 & 0.84 & 0.68 & 0.89 & 0.68 & 0.90 \\
\hline \multicolumn{9}{|l|}{ GK } \\
\hline $\mathbf{A U C}$ & 0.65 & 0.78 & 0.77 & 0.78 & 0.62 & 0.86 & 0.83 & 0.88 \\
\hline cutoff & 0.17 & 1.71 & 1.80 & 1.59 & 0.39 & 0.73 & 0.81 & 0.71 \\
\hline sensitivity & 0.48 & 0.80 & 0.71 & 0.81 & 0.68 & 0.74 & 0.71 & 0.77 \\
\hline specificity & 0.74 & 0.68 & 0.84 & 0.68 & 0.68 & 0.89 & 0.89 & 0.89 \\
\hline
\end{tabular}




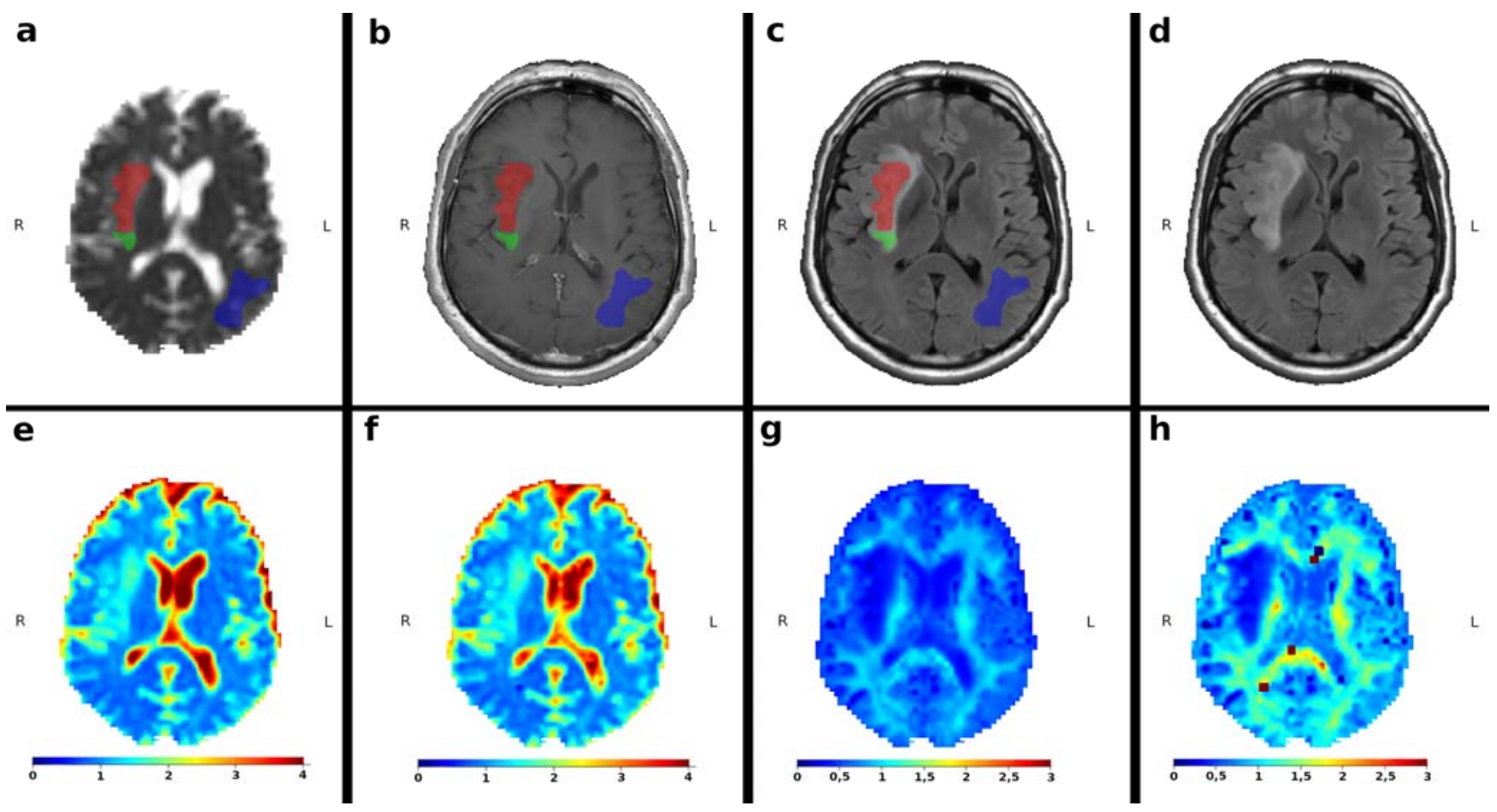

Figure 1 Example of low grade gliomas with the marked regions of interests: red is the tumour, green is the oedema, and blue is the NAWM. All maps are already aligned and interpolated to $\mathrm{T}_{1}$-weighted image. a) mean diffusivity map with masks; b) $\mathrm{T}_{1}$-weighted image with contrast agent; c) $\mathrm{T}_{2}$-weighted image with contrast agent; d) $\mathrm{T}_{2}$-weighted image without contrast agent; e) mean diffusivity map obtained from the conventional kurtosis approach; f) mean diffusivity map obtained from the generalised kurtosis approach; g) mean kurtosis map obtained from the conventional kurtosis approach; h) mean kurtosis map obtained from the generalised kurtosis approach 
medRxiv preprint doi: https://doi.org/10.1101/2020.09.23.20195719; this version posted September 24, 2020. The copyright holder for this preprint (which was not certified by peer review) is the author/funder, who has granted medRxiv a license to display the preprint in perpetuity.

It is made available under a CC-BY-NC-ND 4.0 International license .

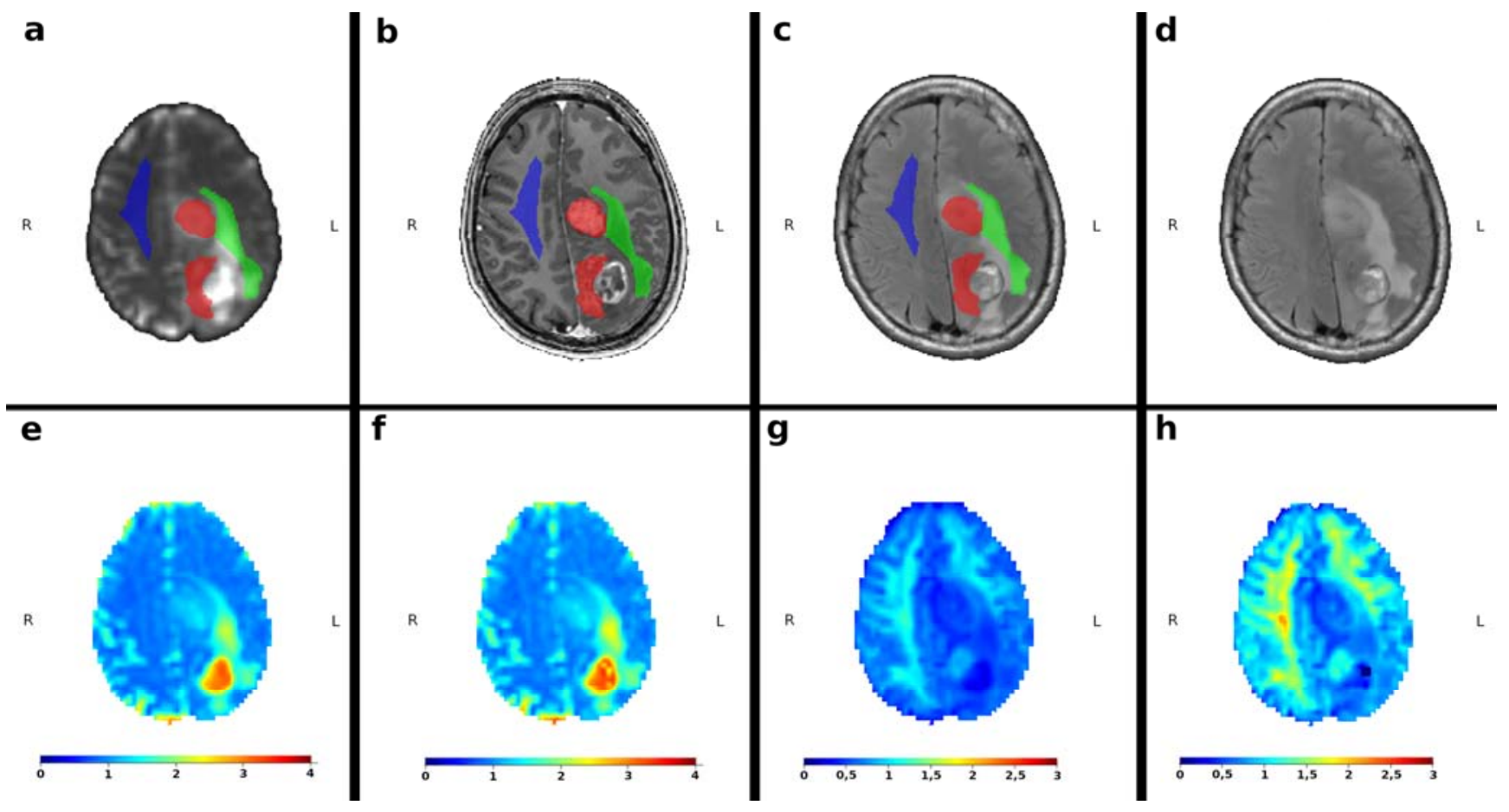

Figure 2 Example of high grade gliomas with the marked regions of interests: red is the tumour, green is the oedema, and blue is the NAWM. All maps are already aligned and interpolated to $\mathrm{T}_{1}$-weighted image. a) mean diffusivity map with masks; b) $\mathrm{T}_{1}$-weighted image with contrast agent; c) $\mathrm{T}_{2}$-weighted image with contrast agent; d) $\mathrm{T}_{2}$-weighted image without contrast agent; e) mean diffusivity map obtained from the conventional kurtosis approach; f) mean diffusivity map obtained from the generalised kurtosis approach; g) mean kurtosis map obtained from the conventional kurtosis approach; h) mean kurtosis map obtained from the generalised kurtosis approach 

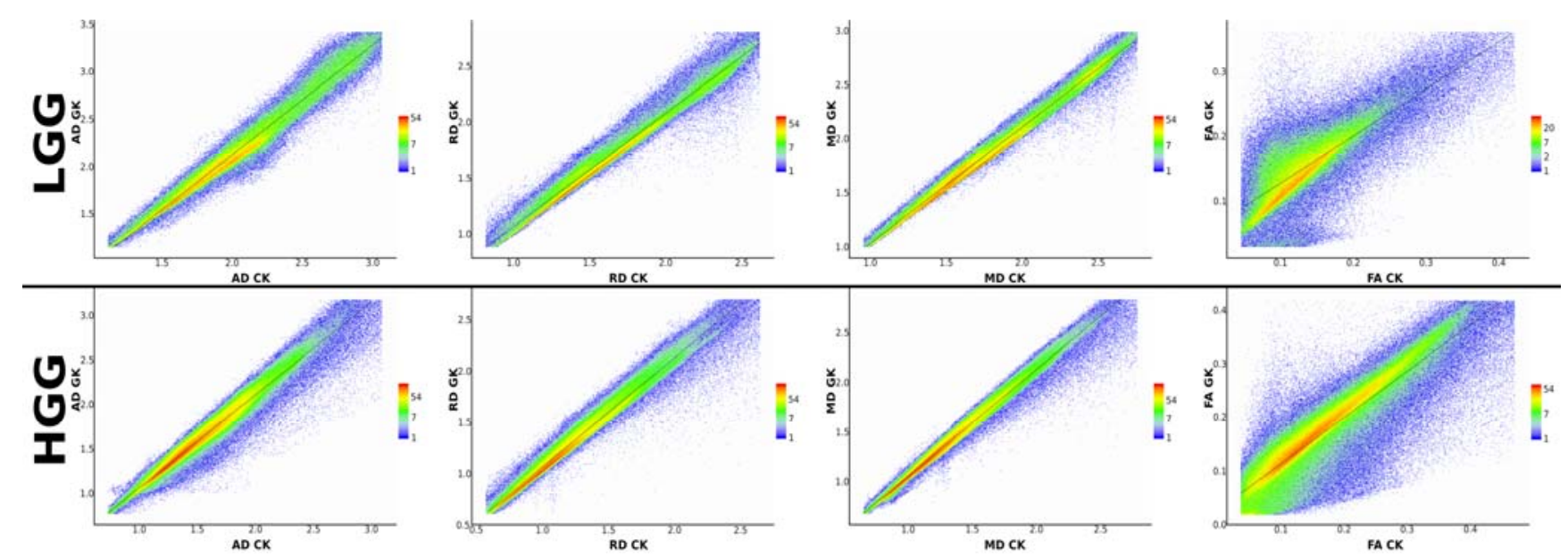

Figure 3 Voxelwise correlations of the diffusion tensor metrics between the conventional and generalised kurtosis approaches. The scalar metrics belong to the tumour masks in low and high grade gliomas for all patients. The columns of plot are axial, radial, and mean diffusivities and fractional anisotropy, respectively

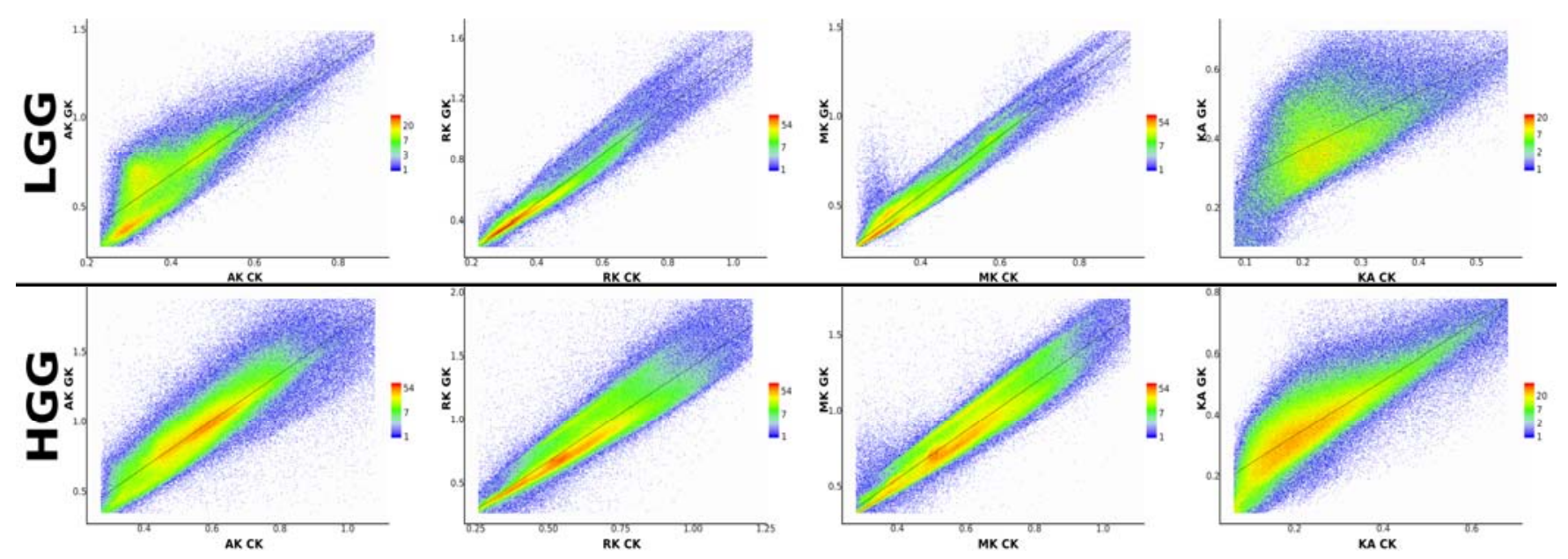

Figure 4 Voxelwise correlations of the diffusion kurtosis metrics between the conventional and generalised kurtosis approaches. The scalar metrics belong to the tumour masks in low and high grade gliomas for all patients. The columns of plot are axial, radial, and mean kurtosis and fractional anisotropy of kurtosis, respectively 

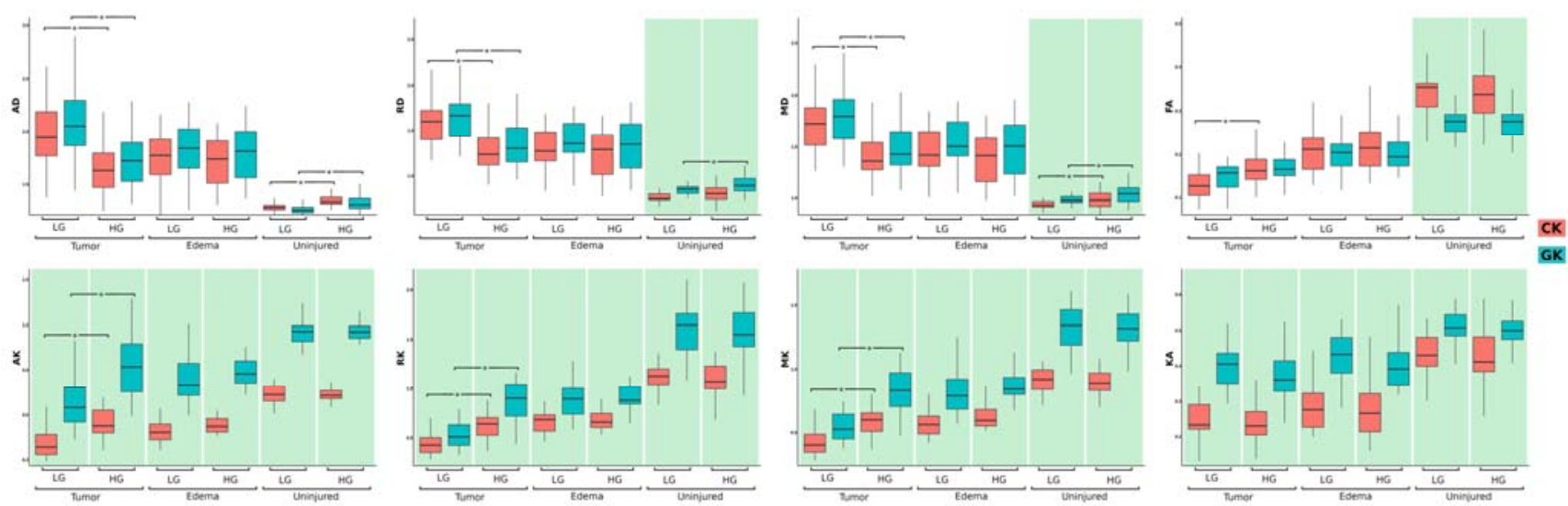

Figure 5 The boxplots of averaged diffusion tensor and kurtosis metrics in different regions: tumour, oedema, and normally appeared white matter. The star-marked brackets exhibit the significant $(\mathrm{p}<$ $0.05)$ difference between low and high grade gliomas for the diffusion metrics. The green background colour emphasise the significant difference between diffusion metrics for the conventional and generalised approaches
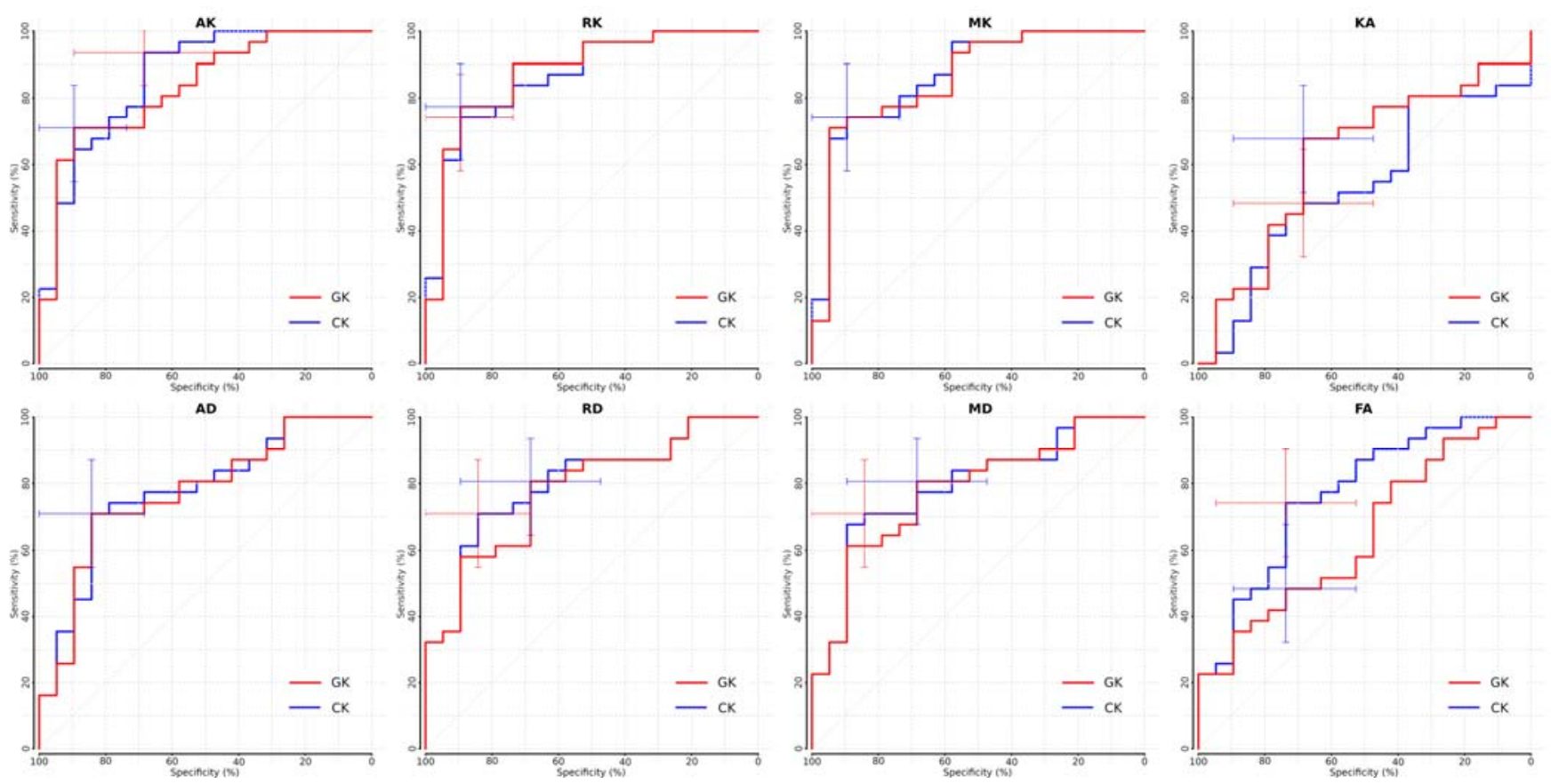

Figure 6 Reciever operating curves (ROC) for the conventional (CK) and generalised kurtosis (GK) approaches for differentiation of low and high grade gliomas. The error-bars exhibits the detected sensitvity and specificty of both methods 
$\mathbf{a}$

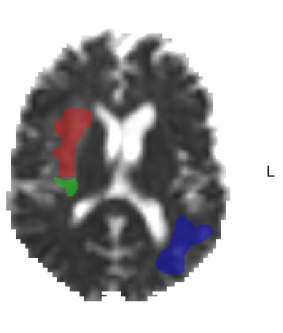

$\mathbf{e}$

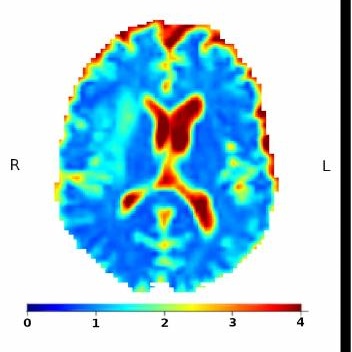

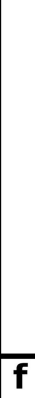

f

C

g

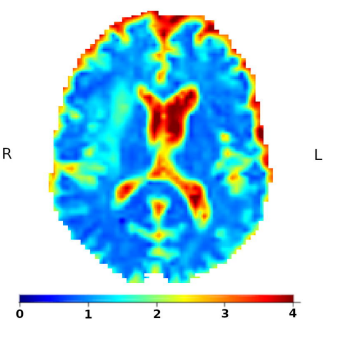

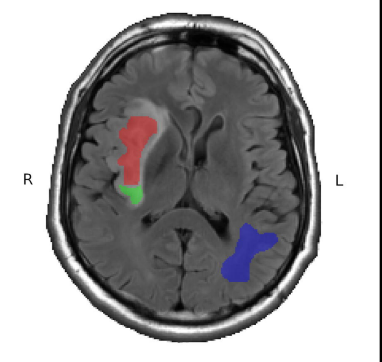

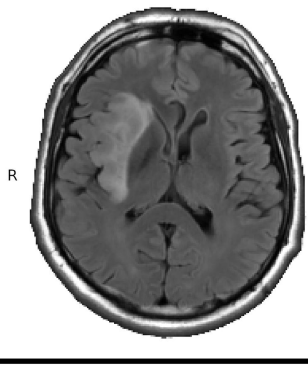

h

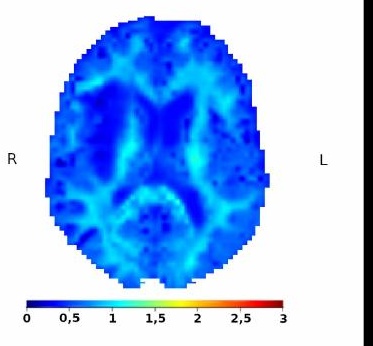

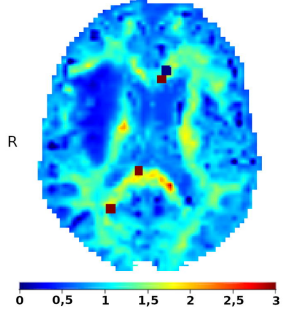


$\mathbf{a}$

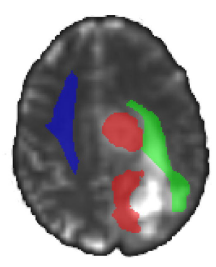

L

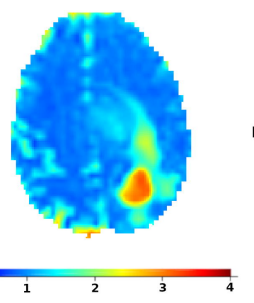

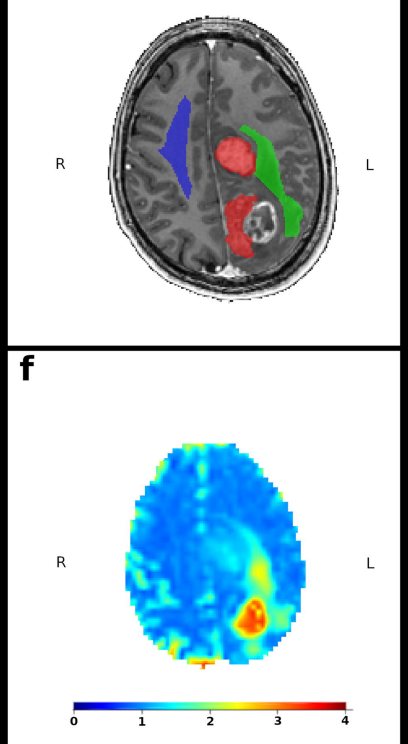

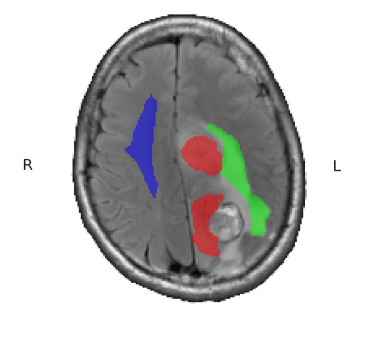

g

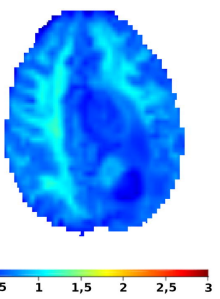

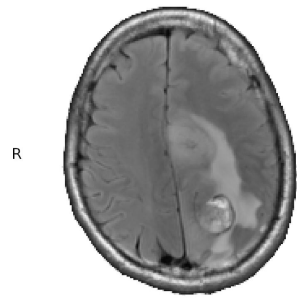

h

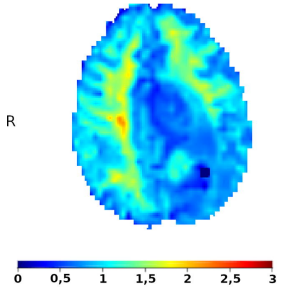




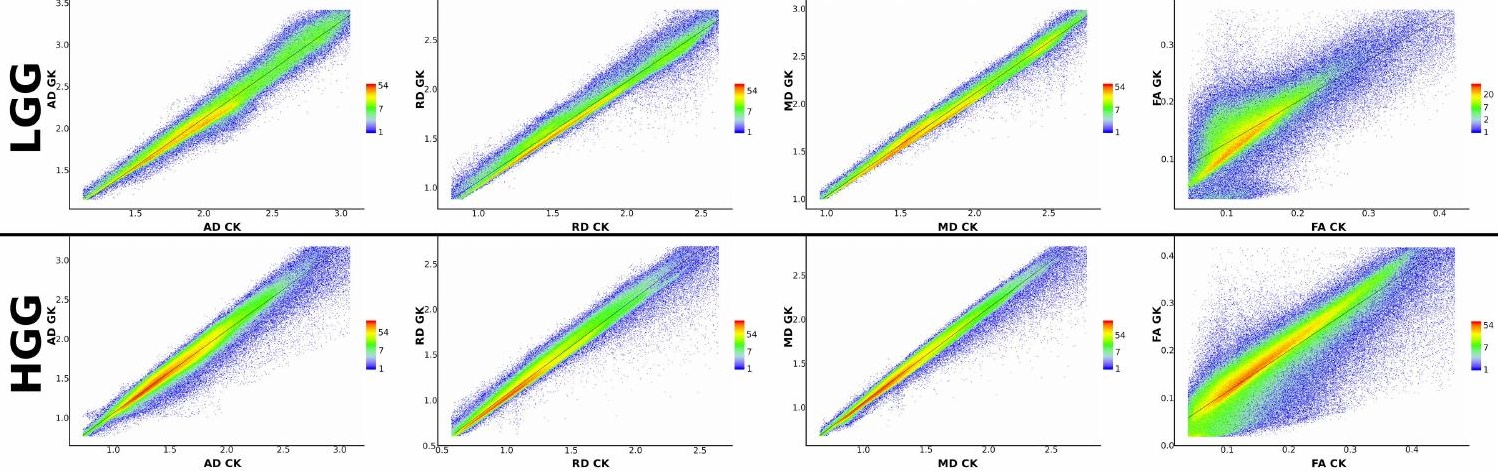




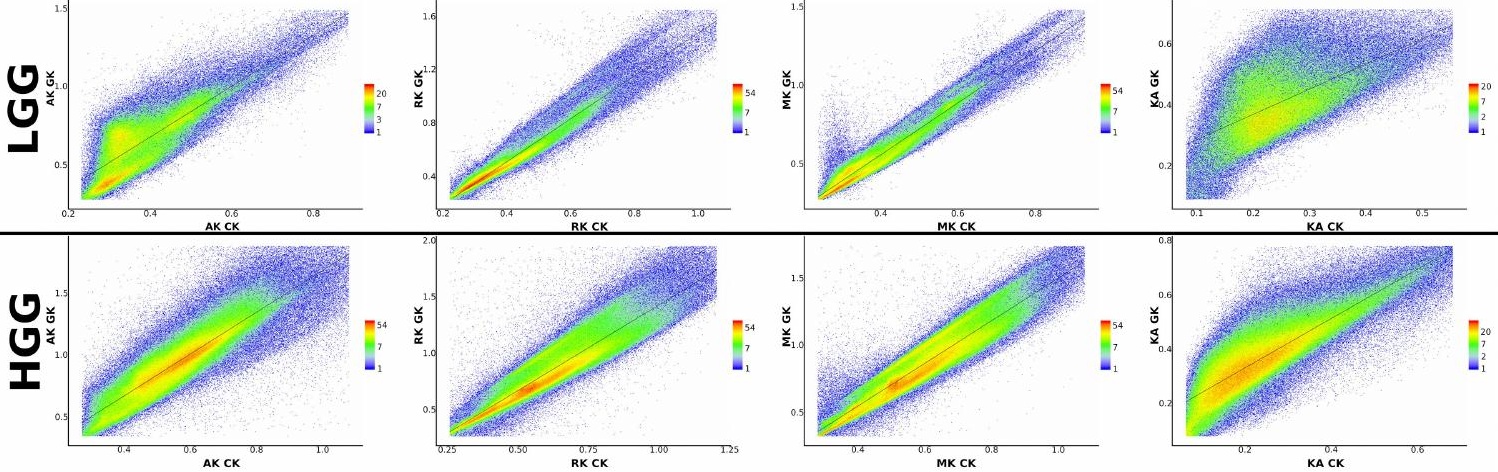




\section{Hath} $+$ C)

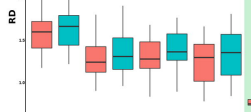

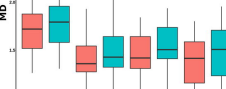

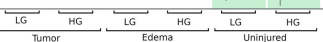

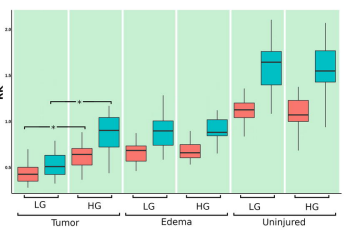
부투부부투

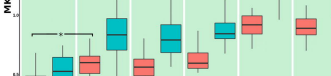

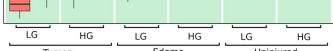

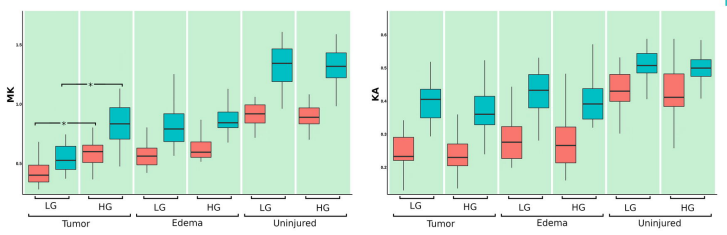




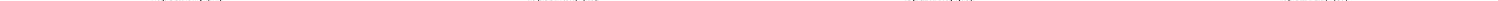

